\title{
Molecular systematics of the genus Pleurotus: analysis of restriction polymorphisms in ribosomal DNA
}

\author{
Béatrice Iraçabal, Georgios Zervakisł and Jacques Labarère
}

Author for correspondence: Jacques Labarère. Tel: +33568431 69. Fax: +33 56843274.

Laboratoire de Génétique

Moléculaire et

d'Amélioration des

Champignons Cultivés,

Université de Bordeaux II INRA, CRA de Bordeaux, BP 81,33883 Villenave d'Ornon Cedex, France

\begin{abstract}
Part of the nuclear ribosomal DNA unit of Pleurotus cornucopiae, including most of the intergenic sequences, was used as a probe to hybridize with DNA from eleven Pleurotus taxa (29 isolates), digested with nine restriction endonucleases. For all digests, a high level of rDNA heterogeneity between and within species was detected, which is in agreement with the phenetic variability already reported in previous studies on Pleurotus taxa. Numerical analysis of the results, performed by use of two different tree-making methods, clearly distinguished among well-defined species, i.e. P. dryinus, $P$. eryngii and $P$. flabellatus, which presented large phenetic distances with the rest of the taxa tested. P. abalonus, despite morphological similarity and interfertility with P. cystidiosus, appears to be a discrete taxon. Although they showed relative affinity, $P$. cornucopiae, $P$. columbinus and $P$. ostreatus presented large distances among their representative isolates, supporting the idea that they correspond to distinct taxa. All strains of $P$. pulmonarius, $P$. sajor-caju and $P$. sapidus having small phenetic distances were closely positioned on dendrograms; in relation to results from recent interfertility and isozyme studies, these results support the common genetic background of $P$. pulmonarius and $P$. sajor-caju; the position of $P$. sapidus remains controversial. Furthermore, the rDNA analysis identified ten fragments suitable for species identification: eight allowed the characterization of five taxa (P. cornucopiae, P. ostreatus, P. sajor-caju, P. abalonus and P. columbinus) and two distinguished among clusters including related taxa. By a combination of just two restriction enzymes, the rDNA probe used seems to be the appropriate tool for both varietal characterization and protection of commercially valuable strains.
\end{abstract}

Keywords: rDNA polymorphism, RFLP, Pleurotus, systematic markers

\section{INTRODUCTION}

The widely distributed fungal genus Pleurotus (Fr.) Quél., order Agaricales (Singer, 1986), is of increasing economic interest (Chang \& Miles, 1991) owing to its ability to readily form fruitbodies of good organoleptic quality on inexpensive agricultural and industrial by-products. In the past most Pleurotus species were dealt with in systematic studies based on morphological and physiological criteria and the outcome of mating tests (Anderson et al., 1973; Eger et al., 1979; Hilber, 1982; Bresinsky et

†Present address: Laboratory of General and Agricultural Microbiology, Agricultural University of Athens, lera Odos 75, 11855 Athens, Greece.

Abbreviation: RFLP, restriction fragment length polymorphism. al., 1987); however the authors failed to arrive at a common conclusion. More recently, mating compatibility studies in strains of the Pleurotus ostreatus complex from North American collections permitted a clear determination of the North American species of oyster mushrooms (Vilgalys et al., 1993). Furthermore, protein electrophoresis and isozyme analysis have been employed to offer more accurate information on the phenetic relationships between and within the Pleurotus species (May \& Royse, 1988; Zervakis \& Labarère, 1992; Zervakis et al., 1994).

Restriction fragment length polymorphisms (RFLPs) of mitochondrial or nuclear rDNA have been adopted for species delineation and phylogenetic analysis in fungal genera such as Aspergillus (Kozlowski \& Stépien, 1982), 
Sclerotinia (Kohn et al., 1988), Phytophthora (Förster et al., 1988), Verticillium (Carder \& Barbara, 1991) and Fusarium (Kistler et al., 1987). Moreover the use of RFLPs in the systematics of basidiomycetes has demonstrated the suitability of this approach for the elucidation of ambiguities in the genera Agaricus (Hintz et al., 1988), Armillaria (Anderson et al., 1987) and Laccaria (Gardes et al., 1991). When using RFLPs in systematics, it is necessary to select a probe which is able to detect phenetic variations in both distant and related species. In fungi, rDNA units are repeated and thus are easily detectable sequences, showing variability at the species level (Walsh et al., 1990; Kohn et al., 1988) and at the strain level (Specht et al., 1984; Cassidy et al., 1984). Indeed, the rDNA unit appears to be especially well suited as a molecular marker because it is composed of conserved gene sequences and variable intergenic sequences which evolve more rapidly than many other parts of the genome (Rogers et al., 1989).

The main aim of this study was to determine whether an RFLP method based on ribosomal polymorphism was suitable for application to the systematics of the genus Pleurotus. Thus, $\mathrm{rDNA}$ variability in 29 isolates belonging to eleven Pleurotus taxa from distant geographic areas was investigated by using nine endonucleases which had either none, one or several sites in the ribosomal probe. Most of the isolates have been already characterized by morphological or physiological criteria (Zervakis \& Balis, 1991) and all by isozyme analyses (Zervakis \& Labarère, 1992; Zervakis $e$ t al., 1994). The resulting RFLP patterns, detected by the $P$. cornucopiae ribosomal probe (composed mainly of coding regions of $18 \mathrm{~S}$ and $5 \mathrm{~S}$ genes, part of the $25 \mathrm{~S}$ gene and two flanking intergenic sequences), were numerically transformed to determine the phenetic distances between strains and the relationships among species. Other goals of this work were to identify molecular markers characteristic of species and to determine whether RFLP could be used in routine examination to identify species, by comparison with results obtained previously using morphological and biochemical criteria.

\section{METHODS}

Strains, media and culture conditions. All fungal isolates used in this study were dikaryotic strains belonging to eleven Pleurotus species (Table 1): P. abalonus (Han et al.), P. columbinus (Quél. apud Bres.) Quél., P. cornucopiae (Paul. ex Pers.) Roll., P. cystidiosus O. K. Miller, $P$. dryinus (Pers. ex Fr.) Kummer, $P$. eryngii (DC. ex Fr.) Quél., $P$. flabellatus (Berck. \& Br.) Sacc., $P$. ostreatus (Jacq. ex Fr.) Kummer, P. pulmonarius (Fr.) Quél., $P$. sajor-caju (Fr.) Sing. and P. sapidus (Schulzer \& Kalchbr.) Sacc. Isolates used in this work included wild strains collected in various geographical areas and three commercial strains (nos 08 , 85 and 90); for isolates no. 04 (P. columbinus) and no. $82(P$. pulmonarius) the wild or commercial origin is unknown (Table 1). Dikaryotic mycelia were cultivated at $25^{\circ} \mathrm{C}$ in the dark in Roux flasks containing liquid Complete Yeast Medium (Raper \& Hoffman, 1974).

Total DNA isolation. Fungal cultures $(15-20 \mathrm{~d}$ old $)$ were harvested by filtration on sterile gauze, rinsed with distilled water and quickly frozen in liquid nitrogen. Total DNA was extracted by using the $N$-cetyl- $N-N-N$-trimethylammonium bromide procedure adapted to basidiomycetes (Noël \& Labarère, 1989).

Restriction enzyme digestion, agarose gel electrophoresis, and Southern blotting. Total DNA was digested singly with nine endonucleases (AvaI, BamHI, ClaI, EcoRI, KpnI, ScaI, SmaI, SstI and HindIII) for $3 \mathrm{~h}$ using 3-4 U ( $\mu \mathrm{g}$ DNA) ${ }^{-1}$ according to the supplier's instructions (Gibco-BRL). Digested DNA fragments were separated by electrophoresis on $0.8 \%$ $(\mathrm{w} / \mathrm{v})$ agarose gels in TEA buffer $(40 \mathrm{mM}$ Tris $/ \mathrm{HCl}, 2 \mathrm{mM}$ EDTA, $20 \mathrm{mM}$ sodium acetate, $\mathrm{pH} 8.0)$ at $60 \mathrm{~V}$ for $12 \mathrm{~h}(30 \mathrm{~cm}$ length) (Maniatis et al., 1982) and transferred onto a nylon membrane (Hybond $\mathrm{N}+$, Amersham) by capillary blotting as described by Southern (1975).

Probe isolation, labelling and hybridizations. The rDNA probe (a pBR322-based recombinant plasmid) is a $6.5 \mathrm{~kb} E c o \mathrm{RI}$ fragment of the rDNA unit of $P$. cornucopiae (Fig. 1) cloned in Eschericbia coli strain JM 83. Plasmid DNA was extracted from liquid bacterial cultures by alkaline lysis according to the method of Vo-Quang et al. (1985). After linearization by HindIII, the probe plasmid was radiolabelled with $25 \mu \mathrm{Ci}\left[\alpha^{32}{ }^{32} \mathrm{P}\right] \mathrm{dCTP}$ [3000 $\mathrm{Ci} \mathrm{mmol}^{-1}\left(111 \mathrm{kBq} \mathrm{mmol}^{-1}\right)$, Amersham] by using the random primer DNA labelling kit (Gibco-BRL) with a specific radioactivity higher than $10^{9}$ c.p.m. $(\mu \mathrm{g} \text { DNA })^{-1}$. Nylon membranes were treated for prehybridization as specified by the manufacturer (Amersham) and hybridized with the rDNA probe at $60^{\circ} \mathrm{C}$ for $12-15 \mathrm{~h}$. Nylon membranes were successively washed in $6 \times$ SSC, $2 \times$ SSC and $0.1 \times$ SSC mixed with $0.1 \%$ $(\mathrm{w} / \mathrm{v})$ SDS for $10-30 \mathrm{~min}$ for each washing step. Autoradiography was performed with Kodak X-Omat AR film at $-70^{\circ} \mathrm{C}$ for $16-24 \mathrm{~h}$ in the presence of an intensifying screen.

Analysis of RFLP data. RFLP analysis was carried out by performing digestion, electrophoresis and Southern hybridizations on total DNA samples from each isolate tested. The molecular size of the detected restriction fragments was evaluated by graphical interpolation using a regression curve, established between the log migration against the log molecular size of lambda DNA digested with HindIII.

To transform the RFLP data for numerical analysis, the molecular phenotypes of each Pleurotus isolate were coded with the aid of a binary system, where the presence (1) and absence (0) of the hybridizing fragments at a particular position on the autoradiograms were scored for each strain. All bands were considered as single fragments unless their relative thickness suggested otherwise. Variations in staining intensity were not taken into account for the construction of the matrix. The measure of distance between isolates was based on the proportion of mismatches among the DNA fragments as follows : $F=1-\left[2 n_{\mathrm{xy}} /\left(n_{\mathrm{x}}+n_{\mathrm{y}}\right)\right]$, where $n_{\mathrm{xy}}$ is the number of fragments in common between two isolates and $n_{\mathrm{x}}, n_{\mathrm{y}}$ are the total number of fragments displayed by each isolate ( $\mathrm{Nei} \& \mathrm{Li}, 1979)$. Then the mean value was calculated for all the tested endonucleases and a distance matrix was established. It should be noted that presence of insertions or deletions, producing length mutation, could disproportionately affect the data analysis.

Dendrograms were constructed, from the distance matrix, with two tree-making methods. The first was the UPGMA (unweighted pair group method using arithmetic means) clustering, which forms groups by successively pairing similar molecular phenotypes according to the magnitude of their observed distances (Sneath \& Sokal, 1973). The software package used was the program NT-SYs (numerical taxonomy system using multivariate statistical programs) on a NixdorfSiemens model PCD-4H computer (Rohlf et al., 1982). The 
Table 1. Pleurotus dikaryons used in this study

\begin{tabular}{|c|c|c|c|c|}
\hline $\begin{array}{c}\text { Isolate code } \\
\text { no. }\end{array}$ & Species & Origin & Type* & Source $\nmid$ \\
\hline 01 & P. abalonus & Japan & Wild & LGMACC 39 \\
\hline 02 & P. abalonus & Japan & Wild & LGMACC 62 \\
\hline 03 & P. columbinus & France & Wild & ATCC 36498 (Vandendries) \\
\hline 04 & P. columbinus & Italy & - & CBS 37351 (Baldacci) \\
\hline 05 & P. cornucopiae & Germany & Wild & ATCC 38547 (Eger) \\
\hline 07 & P. cornucopiae & Netherlands & Wild & CBS 38380 (van der Poel) \\
\hline 08 & P. cornucopiae & France & Commercial & LGMACC 15 \\
\hline 09 & P. cornucopiae & France (Dordogne) & Wild & LGMACC 50 \\
\hline 10 & P. cystidiosus & Greece (Salamina) & Wild & LGAM P50 \\
\hline 11 & P. cystidiosus & USA (Georgia) & Wild & ATCC 28597 (Lombard) \\
\hline 15 & P. dryinus & Germany & Wild & ATCC $36502(\mathrm{Lyr})$ \\
\hline 19 & P. eryngii & Greece (Orestiada) & Wild & LGAM P66 \\
\hline 22 & P. eryngii & Greece (Lasithi) & Wild & LGAM P63 \\
\hline 23 & $P$. eryngii & ex-Czechoslovakia & Wild & ATCC 36047 (Ginterova) \\
\hline 29 & $P$. eryngii & France (Valery sur Somme) & Wild & LGMACC 841043 \\
\hline 34 & P. flabellatus & - & Wild & ATCC 38138 (Graham) \\
\hline 41 & P. ostreatus & Greece (Fthiotida) & Wild & LGAM P60 \\
\hline 45 & P. ostreatus & Germany & Wild & ATCC 32783 (Haider) \\
\hline 59 & P. ostreatus & Spain (Albaladejito) & Wild & LGMACC 871020 \\
\hline 61 & P. ostreatus & France (Gironde) & Wild & LGMACC 861008 \\
\hline 74 & P. pulmonarius & Greece (Vardoussia) & Wild & LGAM P26 \\
\hline 81 & P. pulmonarius & ex-Czechoslovakia & Wild & ATCC 36050 (Ginterova) \\
\hline 82 & P. pulmonarius & USA & - & CBS 13285 (Graauwmans) \\
\hline 85 & P. pulmonarius & Hungary & Commercial & LGMACC 850403 \\
\hline 86 & P. sajor-caju & India & Wild & ATCC 32078 (Rangaswami) \\
\hline 88 & P. sajor-caju & Mauritius & Wild & LGMACC 37 \\
\hline 89 & P. sajor-caju & India & Wild & LGMACC 35 \\
\hline 90 & P. sajor-caju & ex-Czechoslovakia & Commercial & LGMACC 851003 \\
\hline 91 & P. sapidus & USA (Michigan) & Wild & ATCC 24986 (Petersen) \\
\hline
\end{tabular}

*-, Unknown.

† The Pleurotus isolates used in the present study were obtained from the following fungal culture collections: ATCC, American Type Culture Collection (Rockville, USA); CBS, Centraalbureau Voor Schimmelcultures (Baarn, The Netherlands); LGAM, Laboratory of General and Agricultural Microbiology (Agricultural University of Athens, Greece); LGMACC, Laboratoire de Génétique Moléculaire et d'Amélioration des Champignons Cultivés (Université de Bordeaux, II, France).

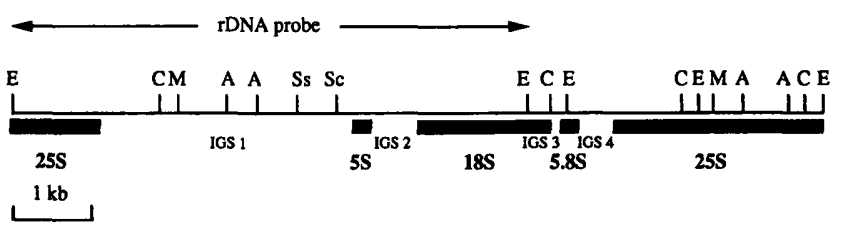

Fig. 1. Restriction map of the cloned $P$. cornucopiae rDNA unit. The sites of the endonucleases used in this work are indicated: A, Aval; C, Clal; E, EcoRl; M, Smal; Sc, Scal and Ss, Sstl. The position of the $6.5 \mathrm{~kb}$ EcoRI insert used as probe is shown.

second was the neighbour-joining method, which finds pairs of molecular phenotypes that minimize the total branch length at each stage of clustering of the phenotypes, starting from a starlike tree (Saitou \& Nei, 1987). Calculations were executed by a computer program developed by Professor J. Sourdis (Department of Genetics, Agricultural University of Athens, Iera Odos 75, 11855 Athens, Greece) with the same hardware.
By reason of the small sample size, the distance values should be considered as rough estimates; nonetheless, as the sample size was similar for all species, the same degree of error should apply. Moreover, the conclusions deriving from RFLP data were compared with previous morphological and biochemical studies performed on the same Pleurotus isolates (Zervakis \& Balis, 1991 ; Zervakis \& Labarère, 1992; Zervakis et al., 1994).

\section{RESULTS}

The rDNA probe was a $6.5 \mathrm{~kb}$ EcoRI insert of the $P$. cornucopiae rDNA unit containing the $5 \mathrm{~S}$ and $18 \mathrm{~S}$ genes, and part of the $25 \mathrm{~S}$ gene flanking two intergenic sequences (IGS 1 and IGS 2) (Fig. 1). Total DNA of the 29 Pleurotus isolates was separately digested by nine endonucleases (each one having a six nucleotide pair recognition sequence): BamHI, HindIII, KpnI, Scal, SmaI, SstI, ClaI, $A v a I$ and EcoRI. After blotting on nylon, restriction digests were hybridized with the radiolabelled rDNA probe. 
Table 2. Binomial matrix of restriction fragments from hybridizations between the rDNA probe of $P$. cornucopiae and total DNA of 29 Pleurotus isolates, digested with nine endonucleases

1, Fragment present; 0 , fragment absent; -, not tested.

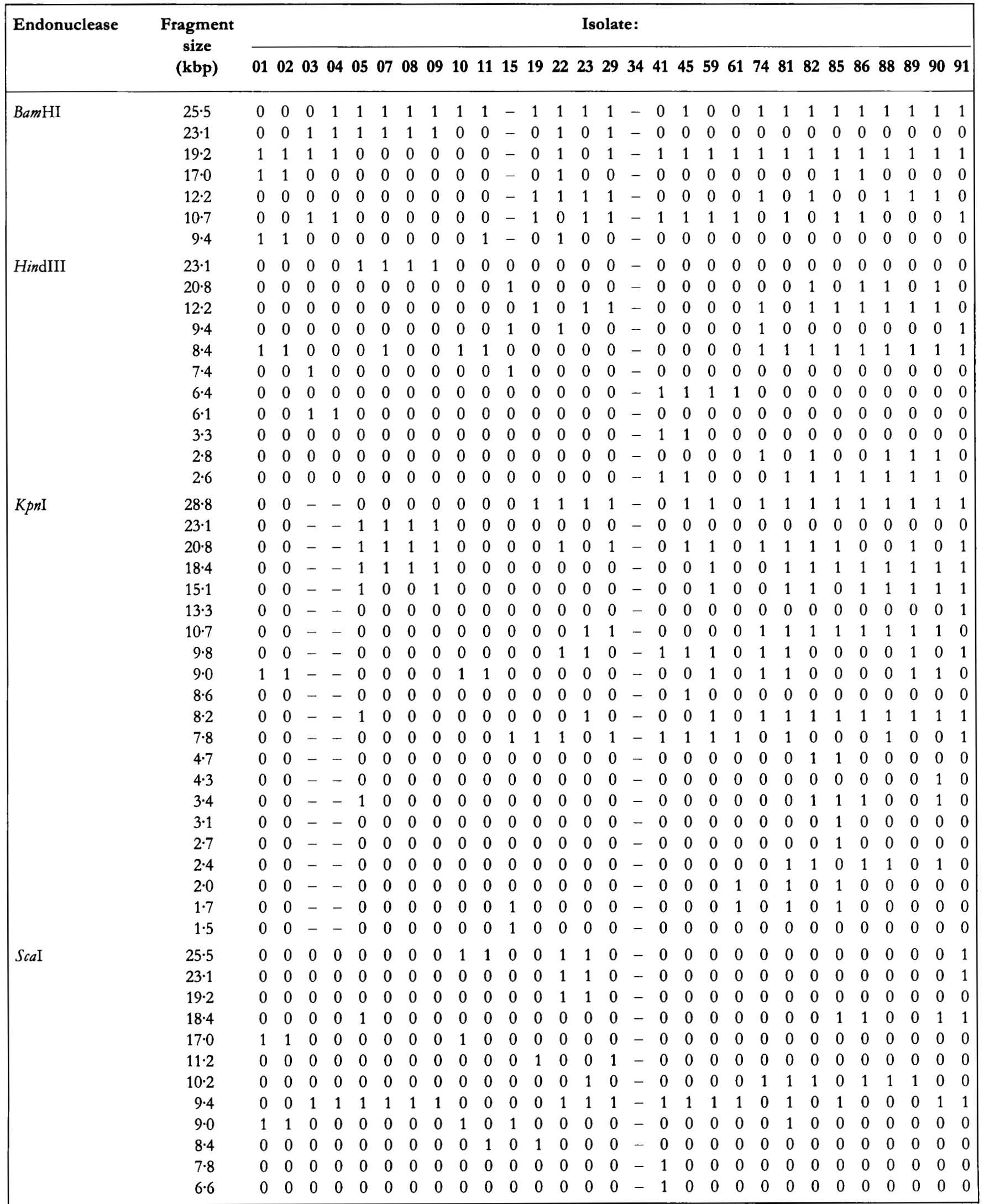


Table 2 (cont.)

\begin{tabular}{|c|c|c|c|c|c|c|c|c|c|c|c|c|c|c|c|c|c|c|c|c|c|c|c|c|c|c|c|c|c|c|}
\hline \multirow[t]{2}{*}{ Endonuclease } & \multirow{2}{*}{$\begin{array}{c}\text { Fragment } \\
\text { size } \\
(\mathbf{k b p})\end{array}$} & \multicolumn{29}{|c|}{ Isolate: } \\
\hline & & 01 & 02 & 03 & 04 & 05 & 07 & 08 & 09 & 10 & 11 & 15 & 19 & 22 & 23 & 29 & 34 & 41 & 45 & 59 & 61 & 74 & 81 & 82 & 85 & 86 & 88 & 89 & 90 & 91 \\
\hline \multirow{15}{*}{$S_{s s t \mathrm{I}}$} & $6 \cdot 2$ & 0 & 0 & 0 & 0 & 0 & 0 & 0 & 0 & 1 & 0 & 0 & 0 & 0 & 0 & 0 & - & 0 & 0 & 0 & 0 & 0 & 0 & 0 & 0 & 0 & 0 & 0 & 0 & 0 \\
\hline & $5 \cdot 9$ & 0 & 0 & 0 & 0 & 0 & 0 & 0 & 0 & 1 & 0 & 0 & 0 & 0 & 0 & 0 & - & 0 & 0 & 0 & 0 & 0 & 0 & 0 & 0 & 0 & 0 & 0 & 0 & 0 \\
\hline & $28 \cdot 8$ & 1 & 1 & 1 & 1 & 0 & 1 & 0 & 0 & 1 & 1 & 1 & 0 & 1 & 0 & 1 & 1 & 0 & 1 & 0 & 0 & 0 & 0 & 1 & 1 & 1 & 1 & - & 1 & - \\
\hline & $25 \cdot 5$ & 0 & 0 & 1 & 0 & 0 & 1 & 0 & 0 & 0 & 0 & 0 & 0 & 1 & 0 & 1 & 0 & 0 & 1 & 1 & 0 & 0 & 0 & 1 & 1 & 1 & 0 & - & 0 & - \\
\hline & $19 \cdot 9$ & 0 & 0 & 1 & 0 & 1 & 1 & 1 & 1 & 0 & 1 & 0 & 0 & 1 & 1 & 1 & 1 & 0 & 1 & 1 & 0 & 0 & 0 & 1 & 1 & 1 & 1 & - & 1 & - \\
\hline & $17 \cdot 7$ & 0 & 0 & 0 & 0 & 0 & 0 & 0 & 0 & 0 & 0 & 0 & 0 & 0 & 0 & 0 & 0 & 0 & 0 & 1 & 1 & 0 & 1 & 0 & 0 & 0 & 0 & - & 0 & - \\
\hline & $16 \cdot 3$ & 0 & 0 & 1 & 0 & 0 & 0 & 0 & 0 & 0 & 0 & 0 & 0 & 0 & 0 & 0 & 0 & 0 & 0 & 0 & 0 & 0 & 0 & 0 & 0 & 0 & 0 & - & 0 & - \\
\hline & $15 \cdot 1$ & 0 & 0 & 0 & 0 & 0 & 0 & 0 & 0 & 0 & 0 & 0 & 0 & 0 & 1 & 0 & 0 & 0 & 0 & 0 & 0 & 0 & 0 & 0 & 0 & 0 & 0 & - & 0 & - \\
\hline & $11 \cdot 7$ & 0 & 0 & 0 & 0 & 0 & 0 & 0 & 0 & 0 & 0 & 0 & 1 & 1 & 0 & 1 & 1 & 0 & 0 & 0 & 0 & 0 & 1 & 1 & 0 & 0 & 0 & - & 0 & - \\
\hline & $10 \cdot 7$ & 0 & 0 & 0 & 0 & 0 & 0 & 0 & 0 & 0 & 0 & 0 & 0 & 0 & 0 & 0 & 0 & 0 & 0 & 0 & 0 & 1 & 1 & 0 & 1 & 1 & 1 & - & 1 & - \\
\hline & $10 \cdot 2$ & 0 & 0 & 0 & 1 & 1 & 1 & 1 & 1 & 0 & 1 & 0 & 0 & 1 & 1 & 1 & 1 & 1 & 1 & 0 & 0 & 0 & 0 & 0 & 0 & 0 & 0 & - & 0 & - \\
\hline & $9 \cdot 4$ & 0 & 0 & 0 & 0 & 0 & 0 & 0 & 0 & 0 & 1 & 0 & 0 & 0 & 0 & 0 & 0 & 0 & 0 & 1 & 1 & 0 & 1 & 0 & 1 & 0 & 1 & - & 0 & - \\
\hline & 8.6 & 0 & 0 & 1 & 1 & 1 & 0 & 1 & 0 & 0 & 0 & 0 & 0 & 1 & 0 & 0 & 0 & 0 & 0 & 0 & 0 & 0 & 0 & 0 & 0 & 0 & 1 & - & 0 & - \\
\hline & $7 \cdot 4$ & 0 & 0 & 0 & 1 & 0 & 1 & 1 & 0 & 0 & 0 & 0 & 0 & 0 & 0 & 0 & 0 & 1 & 1 & 1 & 1 & 0 & 1 & 0 & 0 & 0 & 0 & - & 0 & - \\
\hline & 6.8 & 0 & 0 & 0 & 0 & 0 & 1 & 0 & 0 & 0 & 0 & 0 & 0 & 1 & 0 & 0 & 0 & 0 & 0 & 1 & 0 & 0 & 1 & 1 & 0 & 0 & 1 & - & 0 & - \\
\hline \multirow[t]{19}{*}{ SmaI } & $27 \cdot 0$ & 1 & 1 & 1 & 1 & 1 & 1 & 1 & 0 & 1 & 1 & 1 & 1 & 1 & 1 & 1 & 1 & 0 & 1 & 1 & 1 & 1 & 1 & 1 & 1 & 1 & 1 & 1 & 1 & - \\
\hline & $17 \cdot 7$ & 0 & 0 & 0 & 0 & 1 & 1 & 1 & 0 & 1 & 1 & 1 & 0 & 1 & 1 & 1 & 1 & 1 & 1 & 1 & 1 & 0 & 1 & 0 & 1 & 1 & 1 & 0 & 1 & - \\
\hline & $15 \cdot 7$ & 0 & 0 & 0 & 0 & 0 & 0 & 0 & 0 & 0 & 0 & 0 & 0 & 0 & 0 & 0 & 1 & 0 & 0 & 0 & 0 & 0 & 0 & 0 & 0 & 0 & 0 & 0 & 0 & - \\
\hline & $11 \cdot 7$ & 0 & 0 & 0 & 0 & 0 & 0 & 0 & 0 & 0 & 0 & 0 & 0 & 0 & 0 & 0 & 1 & 0 & 0 & 0 & 0 & 0 & 0 & 0 & 0 & 0 & 0 & 0 & 0 & - \\
\hline & $10 \cdot 7$ & 0 & 0 & 0 & 0 & 0 & 0 & 0 & 0 & 0 & 0 & 1 & 0 & 0 & 0 & 0 & 0 & 0 & 1 & 0 & 0 & 0 & 0 & 0 & 0 & 0 & 0 & 0 & 0 & - \\
\hline & $9 \cdot 8$ & 0 & 0 & 0 & 0 & 0 & 0 & 0 & 0 & 0 & 0 & 1 & 0 & 0 & 0 & 0 & 0 & 0 & 0 & 0 & 0 & 0 & 1 & 1 & 0 & 1 & 1 & 0 & 1 & - \\
\hline & $9 \cdot 4$ & 0 & 0 & 0 & 0 & 0 & 1 & 1 & 1 & 0 & 0 & 0 & 0 & 1 & 0 & 1 & 1 & 0 & 1 & 1 & 1 & 0 & 1 & 0 & 1 & 0 & 0 & 0 & 0 & - \\
\hline & 8.8 & 1 & 1 & 0 & 0 & 0 & 0 & 0 & 0 & 1 & 1 & 0 & 0 & 0 & 0 & 0 & 0 & 0 & 0 & 0 & 0 & 0 & 0 & 0 & 0 & 0 & 0 & 0 & 0 & - \\
\hline & $8 \cdot 6$ & 0 & 0 & 0 & 0 & 0 & 0 & 0 & 0 & 0 & 0 & 0 & 0 & 0 & 0 & 0 & 0 & 0 & 0 & 0 & 0 & 0 & 1 & 1 & 0 & 0 & 0 & 0 & 0 & - \\
\hline & $8 \cdot 2$ & 0 & 0 & 0 & 0 & 0 & 0 & 0 & 0 & 0 & 0 & 0 & 0 & 0 & 0 & 0 & 0 & 0 & 0 & 0 & 0 & 0 & 0 & 0 & 0 & 1 & 1 & 0 & 1 & - \\
\hline & $7 \cdot 8$ & 0 & 0 & 0 & 1 & 0 & 0 & 0 & 0 & 0 & 0 & 0 & 0 & 0 & 0 & 0 & 0 & 1 & 1 & 1 & 1 & 1 & 1 & 0 & 1 & 0 & 0 & 0 & 0 & - \\
\hline & $7 \cdot 2$ & 0 & 0 & 0 & 0 & 0 & 0 & 0 & 0 & 0 & 0 & 0 & 0 & 0 & 0 & 0 & 1 & 0 & 0 & 0 & 0 & 0 & 1 & 0 & 1 & 0 & 0 & 0 & 0 & - \\
\hline & $6 \cdot 6$ & 0 & 0 & 0 & 0 & 0 & 1 & 1 & 1 & 0 & 0 & 0 & 0 & 0 & 0 & 0 & 0 & 0 & 0 & 0 & 0 & 0 & 0 & 0 & 0 & 0 & 0 & 0 & 0 & - \\
\hline & $5 \cdot 6$ & 0 & 0 & 0 & 0 & 0 & 1 & 1 & 0 & 0 & 0 & 0 & 0 & 0 & 0 & 0 & 0 & 0 & 0 & 0 & 0 & 0 & 0 & 0 & 0 & 0 & 0 & 0 & 0 & - \\
\hline & $4 \cdot 8$ & 0 & 0 & 0 & 0 & 0 & 1 & 0 & 0 & 0 & 0 & 0 & 0 & 0 & 0 & 0 & 0 & 0 & 0 & 0 & 0 & 0 & 0 & 0 & 0 & 0 & 0 & 0 & 0 & - \\
\hline & $4 \cdot 5$ & 0 & 0 & 0 & 0 & 0 & 1 & 0 & 0 & 0 & 0 & 0 & 0 & 0 & 0 & 0 & 0 & 0 & 0 & 0 & 0 & 0 & 0 & 0 & 0 & 0 & 0 & 0 & 0 & - \\
\hline & $3 \cdot 8$ & 0 & 0 & 0 & 0 & 0 & 1 & 1 & 1 & 0 & 0 & 0 & 0 & 0 & 0 & 0 & 0 & 0 & 0 & 0 & 0 & 0 & 0 & 0 & 0 & 0 & 0 & 0 & 0 & - \\
\hline & $2 \cdot 9$ & 0 & 0 & 0 & 0 & 0 & 0 & 0 & 0 & 0 & 0 & 0 & 0 & 0 & 0 & 0 & 1 & 0 & 0 & 0 & 0 & 1 & 1 & 1 & 1 & 1 & 1 & 1 & 1 & - \\
\hline & $2 \cdot 3$ & 0 & 0 & 0 & 0 & 0 & 0 & 0 & 0 & 0 & 0 & 0 & 0 & 0 & 0 & 0 & 0 & 1 & 1 & 1 & 1 & 0 & 0 & 0 & 0 & 0 & 0 & 0 & 0 & - \\
\hline \multirow[t]{20}{*}{ AvaI } & $28 \cdot 8$ & 0 & 0 & - & - & 1 & 1 & 1 & 1 & 1 & 0 & 0 & 1 & 1 & 1 & 1 & 1 & 1 & 1 & 1 & 1 & 1 & 1 & 1 & 1 & 1 & 1 & 1 & 1 & 1 \\
\hline & $23 \cdot 1$ & 0 & 0 & - & - & 0 & 0 & 0 & 0 & 0 & 0 & 0 & 0 & 0 & 0 & 1 & 1 & 0 & 0 & 0 & 0 & 0 & 0 & 0 & 0 & 1 & 1 & 0 & 1 & 1 \\
\hline & $19 \cdot 9$ & 0 & 0 & - & - & 1 & 0 & 1 & 1 & 1 & 0 & 0 & 0 & 0 & 0 & 0 & 1 & 0 & 0 & 0 & 0 & 0 & 0 & 1 & 0 & 0 & 1 & 1 & 1 & 0 \\
\hline & $16 \cdot 3$ & 0 & 0 & - & - & 1 & 0 & 0 & 1 & 0 & 0 & 0 & 0 & 0 & 0 & 0 & 1 & 0 & 0 & 0 & 0 & 0 & 1 & 0 & 0 & 0 & 0 & 0 & 0 & 1 \\
\hline & $14 \cdot 5$ & 0 & 0 & - & - & 1 & 1 & 1 & 1 & 0 & 0 & 0 & 0 & 0 & 0 & 0 & 0 & 1 & 1 & 0 & 0 & 0 & 0 & 0 & 0 & 0 & 0 & 0 & 0 & 0 \\
\hline & $10 \cdot 2$ & 0 & 0 & - & - & 1 & 0 & 0 & 1 & 0 & 0 & 0 & 0 & 0 & 0 & 1 & 0 & 0 & 1 & 0 & 0 & 0 & 0 & 0 & 0 & 0 & 0 & 0 & 0 & 0 \\
\hline & $9 \cdot 0$ & 0 & 0 & - & - & 0 & 0 & 0 & 1 & 0 & 0 & 0 & 0 & 0 & 0 & 0 & 1 & 0 & 0 & 0 & 0 & 0 & 0 & 1 & 1 & 1 & 0 & 0 & 1 & 0 \\
\hline & $8 \cdot 4$ & 0 & 0 & - & - & 0 & 0 & 0 & 0 & 0 & 0 & 0 & 0 & 0 & 0 & 0 & 0 & 1 & 0 & 1 & 1 & 1 & 1 & 0 & 1 & 0 & 1 & 1 & 1 & 0 \\
\hline & $7 \cdot 8$ & 0 & 0 & - & - & 0 & 0 & 0 & 0 & 0 & 0 & 0 & 0 & 0 & 0 & 0 & 1 & 0 & 0 & 1 & 1 & 1 & 1 & 1 & 1 & 1 & 0 & 1 & 1 & 1 \\
\hline & $7 \cdot 2$ & 0 & 0 & - & - & 0 & 0 & 0 & 0 & 0 & 0 & 0 & 0 & 0 & 0 & 0 & 1 & 0 & 0 & 1 & 1 & 1 & 0 & 0 & 1 & 0 & 0 & 1 & 0 & 1 \\
\hline & $6 \cdot 3$ & 0 & 0 & - & - & 0 & 0 & 0 & 0 & 0 & 0 & 0 & 0 & 0 & 0 & 0 & 1 & 0 & 0 & 0 & 0 & 0 & 1 & 1 & 0 & 1 & 1 & 1 & 1 & 1 \\
\hline & $6 \cdot 0$ & 0 & 0 & - & - & 0 & 0 & 0 & 0 & 0 & 0 & 0 & 0 & 0 & 0 & 0 & 0 & 0 & 0 & 1 & 1 & 1 & 1 & 0 & 1 & 1 & 1 & 0 & 1 & 0 \\
\hline & $5 \cdot 8$ & 0 & 0 & - & - & 0 & 1 & 1 & 1 & 0 & 0 & 1 & 0 & 0 & 0 & 0 & 0 & 0 & 0 & 0 & 0 & 0 & 0 & 1 & 0 & 0 & 0 & 1 & 0 & 1 \\
\hline & $5 \cdot 5$ & 0 & 0 & - & - & 0 & 0 & 0 & 0 & 0 & 0 & 0 & 0 & 0 & 0 & 0 & 0 & 0 & 0 & 0 & 0 & 0 & 1 & 0 & 1 & 0 & 0 & 0 & 0 & 1 \\
\hline & $4 \cdot 3$ & 0 & 0 & - & - & 0 & 0 & 0 & 0 & 0 & 0 & 1 & 1 & 1 & 1 & 1 & 0 & 1 & 1 & 0 & 1 & 0 & 0 & 0 & 0 & 0 & 0 & 0 & 0 & 0 \\
\hline & $3 \cdot 8$ & 0 & 0 & - & - & 0 & 0 & 0 & 0 & 0 & 0 & 0 & 0 & 0 & 0 & 1 & 0 & 0 & 1 & 0 & 0 & 0 & 0 & 0 & 0 & 0 & 0 & 0 & 0 & 0 \\
\hline & 3.5 & 0 & 0 & - & - & 0 & 0 & 0 & 0 & 0 & 0 & 0 & 0 & 0 & 0 & 0 & 0 & 1 & 0 & 1 & 1 & 0 & 0 & 0 & 0 & 0 & 0 & 0 & 0 & 0 \\
\hline & $3 \cdot 2$ & 0 & 0 & - & - & 0 & 0 & 0 & 0 & 0 & 0 & 0 & 0 & 0 & 0 & 0 & 0 & 0 & 0 & 1 & 0 & 0 & 1 & 1 & 0 & 0 & 0 & 0 & 0 & 0 \\
\hline & $3 \cdot 0$ & 0 & 0 & - & - & 0 & 0 & 0 & 0 & 0 & 0 & 0 & 0 & 0 & 0 & 0 & 0 & 1 & 0 & 0 & 1 & 0 & 0 & 0 & 0 & 0 & 0 & 0 & 0 & 0 \\
\hline & $2 \cdot 7$ & 1 & 1 & - & - & 0 & 1 & 1 & 1 & 0 & 1 & 0 & 0 & 0 & 0 & 0 & 0 & 0 & 0 & 0 & 0 & 0 & 1 & 1 & 0 & 0 & 0 & 0 & 0 & 0 \\
\hline
\end{tabular}


Table 2 (cont.)

\begin{tabular}{|c|c|c|c|c|c|c|c|c|c|c|c|c|c|c|c|c|c|c|c|c|c|c|c|c|c|c|c|c|c|}
\hline \multirow[t]{2}{*}{ Endonuclease } & \multirow{2}{*}{$\begin{array}{c}\text { Fragment } \\
\text { size } \\
(\mathbf{k b p})\end{array}$} & \multicolumn{28}{|c|}{ Isolate: } \\
\hline & & 01 & 02 & 03 & 04 & 050 & 070 & 08 & 09 & 10 & 11 & 15 & 19 & 22 & 23 & 29 & 34 & 41 & 45 & 596 & 617 & 7481 & 182 & 85 & 86 & 88 & 89 & 90 & 91 \\
\hline \multirow{30}{*}{ ClaI } & $2 \cdot 4$ & 0 & 0 & - & - & 0 & 0 & 0 & 0 & 1 & 0 & 0 & 0 & 0 & 0 & 0 & 0 & 0 & 0 & 0 & 0 & 0 & 0 & 0 & 0 & 0 & 0 & 0 & 0 \\
\hline & $2 \cdot 0$ & 0 & 0 & - & - & 0 & 0 & 0 & 0 & 0 & 1 & 0 & 0 & 0 & 0 & 0 & 0 & 0 & 0 & 0 & 0 & 0 & 0 & 0 & 0 & 0 & 0 & 0 & 0 \\
\hline & $1 \cdot 8$ & 0 & 0 & - & - & 0 & 0 & 0 & 0 & 0 & 0 & 0 & 0 & 0 & 0 & 0 & 0 & 1 & 0 & 0 & 0 & 0 & 1 & 1 & 0 & 0 & 0 & 0 & 1 \\
\hline & $1 \cdot 1$ & 0 & 0 & - & - & 0 & 0 & 0 & 0 & 0 & 0 & 0 & 0 & 0 & 0 & 0 & 1 & 0 & 0 & 0 & 0 & 1 & 1 & 1 & 1 & 1 & 1 & 1 & 1 \\
\hline & $1 \cdot 0$ & 0 & 0 & - & - & 0 & 0 & 0 & 0 & 0 & 0 & 0 & 0 & 0 & 0 & 0 & 0 & 1 & 0 & 1 & 1 & 0 & 0 & 0 & 0 & 0 & 0 & 0 & 0 \\
\hline & $24 \cdot 2$ & 1 & 1 & 1 & 1 & 1 & 1 & 1 & 0 & 1 & 1 & 1 & 1 & 1 & 1 & 1 & - & 0 & 1 & 1 & 0 & 0 & 1 & 1 & 1 & 1 & 1 & 1 & 1 \\
\hline & $20 \cdot 8$ & 0 & 0 & 0 & 0 & 1 & 1 & 1 & 1 & 0 & 1 & 0 & 0 & 0 & 1 & 0 & - & 0 & 0 & 0 & 0 & 0 & 0 & 0 & 0 & 0 & 0 & 0 & 0 \\
\hline & $17 \cdot 7$ & 0 & 0 & 0 & 0 & 0 & 1 & 1 & 1 & 0 & 0 & 1 & 1 & 1 & 1 & 1 & - & 0 & 1 & 1 & 1 & 0 & 1 & 1 & 1 & 1 & 0 & 1 & 0 \\
\hline & $16 \cdot 3$ & 0 & 0 & 0 & 0 & 0 & 0 & 0 & 0 & 0 & 0 & 1 & 0 & 0 & 0 & 0 & - & 0 & 0 & 0 & 0 & 0 & 0 & 0 & 0 & 0 & 0 & 0 & 0 \\
\hline & $13 \cdot 3$ & 0 & 0 & 0 & 0 & 0 & 0 & 0 & 0 & 0 & 0 & 0 & 0 & 0 & 0 & 0 & - & 0 & 1 & 1 & 1 & 0 & 0 & 0 & 0 & 0 & 0 & 0 & 0 \\
\hline & $12 \cdot 2$ & 0 & 0 & 0 & 0 & 1 & 1 & 1 & 0 & 0 & 0 & 0 & 0 & 0 & 1 & 0 & - & 0 & 0 & 0 & 0 & 0 & 1 & 0 & 0 & 0 & 0 & 0 & 0 \\
\hline & $9 \cdot 8$ & 0 & 0 & 1 & 1 & 1 & 1 & 1 & 1 & 0 & 0 & 1 & 0 & 0 & 1 & 1 & - & 1 & 1 & 1 & 1 & 1 & 1 & 1 & 1 & 1 & 0 & 1 & 0 \\
\hline & $9 \cdot 2$ & 1 & 1 & 0 & 0 & 0 & 0 & 0 & 0 & 1 & 0 & 0 & 0 & 0 & 0 & 0 & - & 0 & 0 & 0 & 0 & 0 & 1 & 0 & 0 & 0 & 0 & 0 & 0 \\
\hline & 8.8 & 0 & 0 & 0 & 1 & 1 & 1 & 1 & 1 & 0 & 0 & 1 & 0 & 0 & 0 & 1 & - & 0 & 1 & 1 & 1 & 0 & 1 & 1 & 1 & 1 & 0 & 1 & 1 \\
\hline & 8.4 & 1 & 1 & 0 & 0 & 0 & 0 & 0 & 0 & 1 & 1 & 0 & 0 & 0 & 0 & 0 & - & 0 & 0 & 0 & 0 & 0 & 0 & 0 & 0 & 0 & 0 & 0 & 0 \\
\hline & $7 \cdot 8$ & 0 & 0 & 0 & 0 & 0 & 1 & 0 & 0 & 0 & 0 & 0 & 0 & 1 & 1 & 1 & - & 0 & 0 & 0 & 0 & 0 & 0 & 0 & 0 & 0 & 0 & 0 & 0 \\
\hline & 6.8 & 1 & 1 & 1 & 1 & 1 & 1 & 1 & 1 & 1 & 1 & 0 & 1 & 0 & 1 & 0 & - & 0 & 1 & 1 & 1 & 1 & 0 & 1 & 1 & 1 & 1 & 1 & 0 \\
\hline & 6.4 & 1 & 1 & 0 & 0 & 1 & 1 & 1 & 1 & 1 & 1 & 1 & 1 & 1 & 0 & 0 & - & 0 & 1 & 0 & 1 & 0 & 0 & 1 & 0 & 0 & 0 & 0 & 1 \\
\hline & 6.0 & 0 & 0 & 0 & 1 & 0 & 0 & 0 & 0 & 0 & 0 & 0 & 0 & 0 & 0 & 0 & - & 0 & 0 & 1 & 1 & 0 & 0 & 0 & 0 & 0 & 0 & 0 & 0 \\
\hline & $5 \cdot 2$ & 0 & 0 & 0 & 0 & 1 & 1 & 1 & 1 & 0 & 1 & 0 & 0 & 0 & 1 & 0 & - & 0 & 0 & 0 & 0 & 0 & 0 & 0 & 0 & 0 & 0 & 0 & 0 \\
\hline & $4 \cdot 4$ & 0 & 0 & 0 & 0 & 0 & 0 & 0 & 0 & 0 & 0 & 0 & 0 & 1 & 0 & 0 & - & 0 & 1 & 1 & 1 & 0 & 0 & 0 & 0 & 0 & 0 & 0 & 0 \\
\hline & $3 \cdot 8$ & 0 & 0 & 1 & 1 & 0 & 0 & 0 & 0 & 0 & 0 & 0 & 0 & 1 & 1 & 0 & - & 1 & 1 & 1 & 1 & 0 & 0 & 0 & 0 & 0 & 0 & 0 & 0 \\
\hline & 3.5 & 0 & 0 & 0 & 0 & 1 & 1 & 1 & 1 & 0 & 0 & 0 & 0 & 0 & 0 & 0 & - & 0 & 0 & 0 & 0 & 0 & 0 & 0 & 0 & 0 & 0 & 0 & 0 \\
\hline & $3 \cdot 2$ & 0 & 0 & 0 & 1 & 0 & 0 & 0 & 0 & 0 & 1 & 0 & 0 & 1 & 1 & 0 & - & 0 & 0 & 0 & 1 & 0 & 0 & 0 & 0 & 0 & 0 & 0 & 0 \\
\hline & $2 \cdot 7$ & 0 & 0 & 0 & 1 & 1 & 1 & 1 & 1 & 0 & 1 & 0 & 1 & 1 & 1 & 0 & - & 1 & 1 & 1 & 1 & 0 & 0 & 0 & 0 & 0 & 0 & 0 & 0 \\
\hline & $2 \cdot 2$ & 0 & 0 & 1 & 1 & 1 & 1 & 1 & 1 & 0 & 0 & 0 & 1 & 1 & 1 & 0 & - & 1 & 1 & 1 & 1 & 0 & 0 & 0 & 0 & 0 & 0 & 0 & 0 \\
\hline & 1.9 & 0 & 0 & 0 & 0 & 1 & 1 & 1 & 1 & 0 & 1 & 0 & 0 & 0 & 0 & 0 & - & 1 & 0 & 0 & 0 & 0 & 0 & 0 & 0 & 0 & 0 & 0 & 0 \\
\hline & $1 \cdot 1$ & 0 & 0 & 0 & 0 & 1 & 1 & 1 & 0 & 0 & 0 & 0 & 0 & 0 & 0 & 0 & - & 0 & 0 & 0 & 0 & 0 & 0 & 0 & 0 & 0 & 0 & 0 & 0 \\
\hline & 0.7 & 0 & 0 & 0 & 0 & 1 & 1 & 1 & 0 & 0 & 0 & 0 & 0 & 0 & 0 & 0 & - & 0 & 0 & 0 & 0 & 0 & 0 & 0 & 0 & 0 & 0 & 0 & 0 \\
\hline & 0.6 & 0 & 0 & 0 & 1 & 0 & 0 & 0 & 0 & 0 & 0 & 0 & 0 & 1 & 1 & 1 & - & 1 & 1 & 0 & 0 & 0 & 0 & 0 & 0 & 0 & 0 & 0 & 0 \\
\hline \multirow[t]{24}{*}{ EcoRI } & $25 \cdot 5$ & 1 & 0 & 1 & 1 & 1 & 1 & 0 & 0 & 1 & 1 & 0 & 0 & 1 & 0 & 1 & 0 & 0 & 1 & 0 & 0 & 1 & 1 & 1 & 0 & 0 & 1 & 1 & 0 \\
\hline & $19 \cdot 9$ & 1 & 0 & 1 & 0 & 0 & 0 & 0 & 0 & 1 & 0 & 1 & 1 & 1 & 1 & 1 & 0 & 0 & 1 & 1 & 0 & 11 & 1 & 1 & 1 & 0 & 1 & 1 & 0 \\
\hline & $17 \cdot 7$ & 0 & 0 & 0 & 0 & 0 & 0 & 0 & 0 & 0 & 0 & 1 & 0 & 0 & 0 & 0 & 1 & 0 & 0 & 0 & 0 & $\begin{array}{ll}1 & 1\end{array}$ & 1 & 1 & 1 & 0 & 1 & 0 & 0 \\
\hline & $17 \cdot 0$ & 0 & 0 & 0 & 0 & 0 & 0 & 0 & 0 & 0 & 0 & 0 & 0 & 1 & 1 & 0 & 0 & 0 & 0 & 0 & 0 & 0 & 0 & 0 & 0 & 0 & 0 & 0 & 0 \\
\hline & $15 \cdot 7$ & 0 & 0 & 0 & 0 & 1 & 1 & 1 & 0 & 0 & 0 & 0 & 0 & 0 & 0 & 0 & 1 & 0 & 0 & 0 & 0 & 0 & 0 & 0 & 0 & 0 & 0 & 0 & 0 \\
\hline & $10 \cdot 2$ & 0 & 0 & 0 & 0 & 0 & 0 & 0 & 0 & 0 & 0 & 1 & 0 & 0 & 0 & 0 & 0 & 0 & 0 & 0 & 0 & 0 & 0 & 0 & 0 & 0 & 0 & 0 & 0 \\
\hline & $9 \cdot 4$ & 0 & 0 & 1 & 0 & 1 & 1 & 1 & 1 & 0 & 1 & 1 & 0 & 1 & 1 & 0 & 1 & 0 & 1 & 0 & 0 & 1 & 0 & 1 & 0 & 0 & 0 & 0 & 1 \\
\hline & $8 \cdot 2$ & 0 & 0 & 1 & 1 & 1 & 1 & 1 & 1 & 0 & 0 & 0 & 0 & 0 & 0 & 0 & 0 & 0 & 0 & 0 & 0 & 0 & 0 & 0 & 0 & 0 & 0 & 0 & 0 \\
\hline & $8 \cdot 0$ & 0 & 0 & 0 & 0 & 0 & 0 & 0 & 0 & 0 & 0 & 1 & 0 & 0 & 0 & 0 & 1 & 0 & 0 & 0 & 0 & 1 & 1 & 1 & 1 & 0 & 1 & 1 & 1 \\
\hline & $7 \cdot 6$ & 0 & 0 & 0 & 0 & 0 & 0 & 0 & 0 & 0 & 1 & 0 & 0 & 0 & 0 & 0 & 0 & 0 & 0 & 0 & 0 & 0 & 0 & 0 & 0 & 0 & 0 & 0 & 0 \\
\hline & $7 \cdot 2$ & 0 & 0 & 0 & 0 & 0 & 1 & 0 & 0 & 0 & 0 & 1 & 1 & 0 & 1 & 0 & 1 & 0 & 0 & 0 & 0 & 0 & 0 & 0 & 1 & 0 & 1 & 1 & 0 \\
\hline & 6.8 & 0 & 0 & 1 & 1 & 1 & 1 & 1 & 1 & 0 & 0 & 0 & 0 & 1 & 0 & 0 & 0 & 1 & 1 & 1 & 1 & 1 & 1 & 0 & 1 & 0 & 1 & 1 & 1 \\
\hline & 6.4 & 1 & 1 & 0 & 0 & 0 & 0 & 0 & 0 & 0 & 1 & 0 & 0 & 0 & 0 & 0 & 0 & 0 & 0 & 0 & 0 & 0 & 0 & 0 & 0 & 0 & 0 & 0 & 0 \\
\hline & $6 \cdot 1$ & 0 & 0 & 0 & 0 & 0 & 0 & 0 & 0 & 0 & 0 & 0 & 0 & 0 & 0 & 0 & 0 & 0 & 0 & 0 & 0 & 0 & 1 & 1 & 0 & 0 & 0 & 0 & 0 \\
\hline & $5 \cdot 8$ & 0 & 0 & 0 & 0 & 0 & 0 & 0 & 0 & 0 & 0 & 0 & 0 & 0 & 0 & 0 & 0 & 0 & 0 & 0 & 0 & 0 & 1 & 1 & 0 & 0 & 0 & 0 & 0 \\
\hline & $5 \cdot 0$ & 0 & 0 & 0 & 0 & 0 & 0 & 0 & 0 & 0 & 0 & 0 & 0 & 0 & 0 & 0 & 0 & 0 & 0 & 0 & 0 & 1 & 1 & 1 & 1 & 1 & 1 & 1 & 0 \\
\hline & $4 \cdot 6$ & 0 & 1 & 1 & 0 & 0 & 0 & 0 & 0 & 0 & 0 & 0 & 0 & 0 & 0 & 0 & 0 & 0 & 0 & 0 & 0 & 0 & 0 & 0 & 0 & 0 & 0 & 0 & 0 \\
\hline & $4 \cdot 5$ & 0 & 0 & 0 & 0 & 0 & 0 & 0 & 0 & 0 & 0 & 0 & 0 & 0 & 0 & 0 & 0 & 0 & 0 & 0 & 0 & 0 & 0 & 0 & 1 & 1 & 1 & 1 & 0 \\
\hline & $4 \cdot 2$ & 0 & 0 & 1 & 0 & 0 & 0 & 0 & 0 & 0 & 0 & 0 & 0 & 0 & 0 & 0 & 0 & 0 & 0 & 0 & 0 & 1 & 1 & 0 & 0 & 0 & 0 & 0 & 0 \\
\hline & 3.8 & 0 & 0 & 0 & 0 & 0 & 0 & 0 & 0 & 0 & 0 & 0 & 0 & 0 & 0 & 0 & 0 & 0 & 0 & 0 & 0 & 0 & 0 & 0 & 0 & 1 & 0 & 1 & 1 \\
\hline & $3 \cdot 6$ & 1 & 1 & 0 & 0 & 0 & 0 & 0 & 0 & 0 & 0 & 0 & 0 & 0 & 0 & 0 & 0 & 0 & 0 & 0 & 0 & 0 & 0 & 1 & 1 & 1 & 1 & 1 & 1 \\
\hline & $3 \cdot 3$ & 1 & 1 & 0 & 0 & 0 & 0 & 0 & 0 & 0 & 0 & 0 & 0 & 0 & 0 & 0 & 0 & 0 & 0 & 0 & 0 & 0 & 1 & 1 & 0 & 0 & 0 & 0 & 0 \\
\hline & 3.0 & 1 & 1 & 0 & 0 & 0 & 0 & 0 & 0 & 0 & 0 & 0 & 0 & 0 & 0 & 0 & 0 & 0 & 0 & 0 & 0 & 0 & 0 & 0 & 0 & 0 & 0 & 0 & 0 \\
\hline & $2 \cdot 7$ & 1 & 1 & 0 & 0 & 0 & 0 & 0 & 0 & 0 & 0 & 0 & 0 & 0 & 0 & 0 & 0 & 0 & 0 & 0 & 0 & 0 & 0 & 0 & 0 & 0 & 0 & 0 & 0 \\
\hline
\end{tabular}


(a)

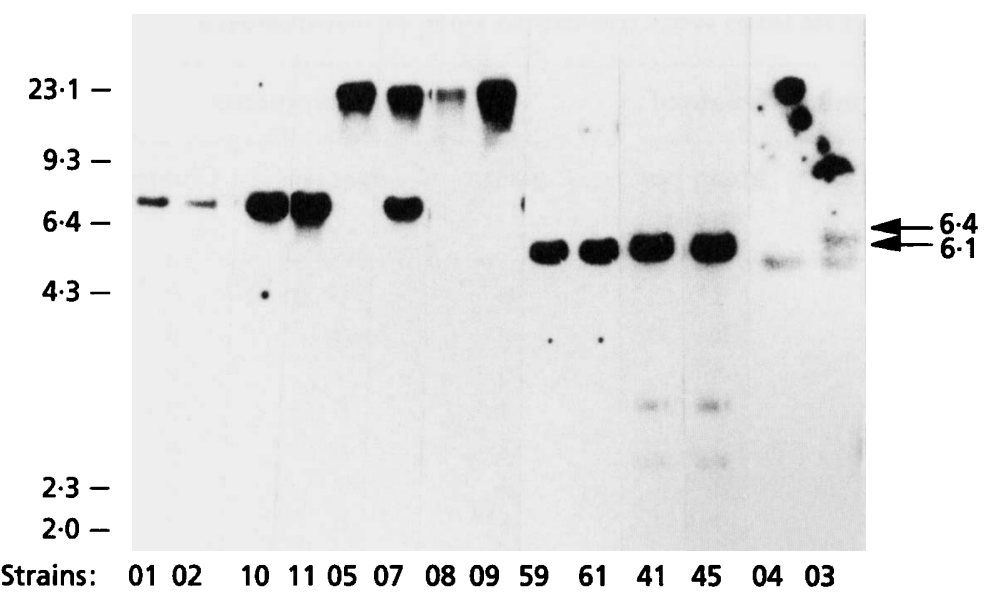

(b)

(c)

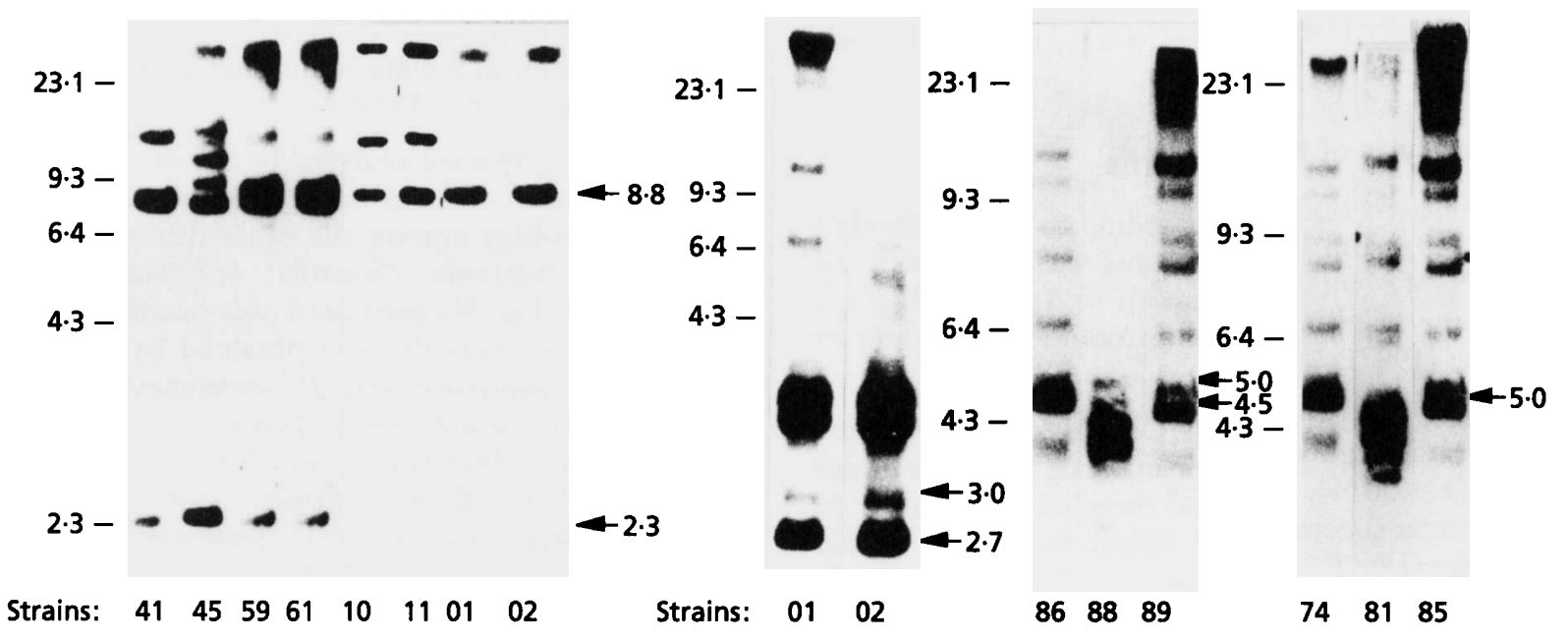

Fig. 2. Representative RFLP patterns produced by hybridization of Pleurotus DNA with the rDNA probe. Total DNA was digested by HindIII (a), Smal (b), and EcoRI (c). Strains are numbered as in Table 1. Fragment sizes are in kb.

To study the rDNA polymorphism, the RFLP fragments were examined on the basis of (i) their strain specificity by comparison of patterns obtained for the nine endonucleases tested, (ii) their similarity between isolates within a single species and (iii) their relatedness among different species. The resulting RFLP autoradiograms were used to construct a matrix of phenetic distances, generated by the method of Nei \& Li (1979), which was then computed to produce the dendrograms. Furthermore, RFLP patterns were compared in order to find whether some RFLP fragments could characterize a species or a cluster of species and might therefore be suitable as systematic markers.

\section{The rDNA polymorphism within the genus Pleurotus}

Hybridizations of the $P$. cornucopiae rDNA probe with total DNA of the 29 Pleurotus isolates revealed 160 fragments showing 186 RFLP patterns after performing a total of nine digestions (Table 2); representative hybridization patterns are presented in Fig. 2. When all the digestions were considered, each of the 29 isolates studied possessed a characteristic pattern. The highest number of bands (21, 24, 25 and 26) was obtained when total DNA was digested by $A v a \mathrm{I}, \mathrm{Eco \textrm {RI }}, K p n \mathrm{I}$ or ClaI (Table 3). The highest degree of discrimination was obtained with digestions by $A v a \mathrm{I}, C l a \mathrm{I}$, EcoRI, KpnI or SstI. The BamHI, HindIII, SmaI and ScaI digestions appeared to be less polymorphic, thus forming numerous groups of strains sharing the same pattern. SstI was the only endonuclease which presented entirely unique phenotypes among the Pleurotus strains examined (with the sole exception of $P$. abalonus isolates), although it produced a relatively low total number of DNA fragments. In the same way, when total DNA was digested by EcoRI, 25 among the 29 isolates studied possessed different patterns. The EcoRI-unidentified isolates 41 and 61 on the one hand and 10 and 29 on the other hand could 
Table 3. Restriction sites detected within the rDNA probe and numbers of RFLP fragments obtained from 29 Pleurotus isolates with the use of nine endonucleases

\begin{tabular}{|c|c|c|c|c|c|c|}
\hline \multirow[t]{2}{*}{ Enzyme } & \multirow{2}{*}{$\begin{array}{c}\text { Restriction } \\
\text { sites in } \\
\text { the probe }\end{array}$} & \multicolumn{2}{|c|}{ Fragments obtained } & \multicolumn{3}{|c|}{ Specific fragments } \\
\hline & & Total & $\begin{array}{l}\text { Mean per } \\
\text { strain }\end{array}$ & Strain & Species & Cluster \\
\hline $\mathrm{BamHI}$ & 0 & 7 & 3 & 0 & 0 & 0 \\
\hline HindIII & 0 & 11 & 2 & 0 & 3 & 0 \\
\hline$K p n \mathbf{I}$ & 0 & 21 & 5 & 6 & 1 & 0 \\
\hline ScaI & 1 & 14 & 2 & 4 & 0 & 0 \\
\hline SstI & 1 & 13 & $3-4$ & 2 & 0 & 0 \\
\hline SmaI & 1 & 19 & $3-4$ & 4 & 1 & 1 \\
\hline ClaI & 1 & 25 & $7-8$ & 1 & 0 & 0 \\
\hline AvaI & 2 & 26 & 6 & 2 & 0 & 0 \\
\hline EcoRI & 2 & 24 & $5-6$ & 2 & 3 & 1 \\
\hline
\end{tabular}

be distinguished by HindIII, KpnI, ScaI, SstI, SmaI, AvaI or ClaI digestions.

\section{Numerical analysis of RFLP patterns}

The distance matrix obtained according to the method of Nei \& $\mathrm{Li}$ (1979) from RFLP patterns was then analysed either by UPGMA with the program NT-sYs (Rohlf et al., 1982 ) or by the neighbour-joining method according to Saitou \& Nei (1987).

On the dendrogram obtained by UPGMA (Fig. 3a) nine clusters were distinguished at a distance level of more than $50 \%(D>50 \%)$. Seven of them corresponded to separate Pleurotus species, i.e. P. flabellatus (A), P. dryinus (B), P. sapidus (D), P. cornucopiae (F), P. eryngii (G), $P$. columbinus $(\mathrm{H})$ and $P$. ostreatus $(\mathrm{I})$. The other two clusters included two species each: $P$. cystidiosus $-P$. abalonus (cluster $\mathrm{C}$ ) and $P$. pulmonarius-P. sajor-caju (cluster E).

Among the eleven species, $P$. flabellatus and $P$. dryinus, which presented distance values well over $75 \%$ in all comparisons, appeared clearly distant from other species. Isolates belonging to $P$. columbinus and $P$. cornucopiae formed tight intraspecific groups $(D<30 \%$ in each case, the French strains of $P$. cornucopiae being in close proximity: $D=15 \%$ ), whereas $P$. ostreatus isolates seemed to be less related $(D=18-42 \%)$. Strains of $P$. eryngii, despite their placement into a unique cluster, had relatively large distances between them $[D=41-63 \%$; isolates from northeastern Greece (no 19) and Crete (no 22) being the most distant].

$P$. ostreatus and $P$. columbinus had closer interspecific relationships, and this was also the case with $P$. sapidus (cluster D) and the compound cluster of $P$. pulmonarius $-P$. sajor-caju (cluster E). In cluster E, strains appeared to be particularly related, although originally assigned to different species, as their distance values did not exceed $55 \%$. Similarly, strains of cluster $C(P$. cystidiosus and $P$. abalonus) showed an increased affinity $(D=27-45 \%)$, and seemed to be well discriminated from the other clusters $(D>60 \%)$; the two isolates of $P$. abalonus having the tightest bonds $(D=3 \%)$ among all strains examined; while $P$. cystidiosus isolates exhibited higher intraspecific distance values $(D<35 \%)$.

When the results were analysed by the neighbour-joining method, which is considered the best for obtaining accurate topology among the other distance matrix and parsimony methods (Sourdis \& Nei, 1988), the dendrogram (Fig. 3b) presented only one major difference in comparison with the one obtained by UPGMA: $P$. ostreatus, $P$. columbinus and $P$. cornucopiae isolates, previously separated into three clusters, were joined into a common larger cluster, $P$. ostreatus and $P$. columbinus being the most related. Relative positions of all other strains remained identical, notably $P$. pulmonarius $-P$. sajor-caju forming one cluster as well as $P$. abalonus and $P$. cystidiosus.

\section{RFLP fragments usable as molecular markers for Pleurotus species identification}

In order to obtain molecular markers for characterization of Pleurotus species, a detailed analysis of patterns was carried out to find RFLP fragments which allowed single species or clusters of species to be distinguished.

In the composite clusters, the $8.8 \mathrm{~kb} \mathrm{SmaI} \mathrm{fragment} \mathrm{was}$ exclusively present in strains of cluster $C(P$. abalonus and $P$. cystidiosus); the $5 \mathrm{~kb}$ EcoRI fragment was specifically detected in strains of cluster $\mathrm{E}(P$. pulmonarius and $P$. sajorcaju). Eight species-specific fragments allowed differentiation among five species. All the $P$. ostreatus isolates examined shared the $6.4 \mathrm{~kb}$ HindIII and $2.3 \mathrm{~kb} S m a \mathrm{I}$ bands, while strains of $P$. corncucopiae could be characterized by the $23.1 \mathrm{~kb}$ HindIII and $23.1 \mathrm{~kb} K p n \mathrm{I}$ fragments, and $P$. sajor-caju by the $4.5 \mathrm{~kb}$ EcoRI band. Likewise, $P$. columbinus could be characterized by the $6.1 \mathrm{~kb}$ HindIII fragment and $P$. abalonus by the $2.7 \mathrm{~kb}$ and $3.0 \mathrm{~kb}$ EcoRI fragments.

In the search for patterns specific to a cluster, it is worth noting that $P$. abalonus isolates showed interspecies affinities with $P$. cystidiosus by featuring common patterns

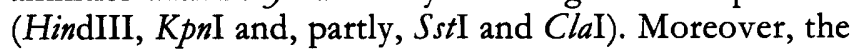


(a)

P. ostreatus
P. columbinus
P. eryngii
P. cornucopiae
P. sajor-caju
P. pulmonarius
P. sajor-caju
P. pulmonarius
P. sapidus
P. abalonus
P. cystidiosus
P. dryinus
P. flabellatus

(b)

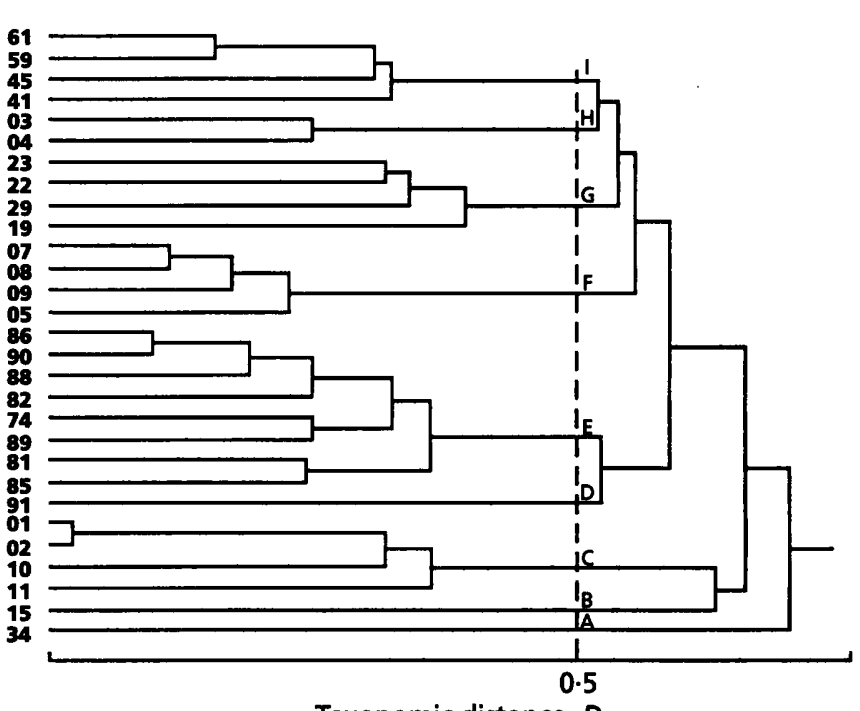

Taxonomic distance, $D$

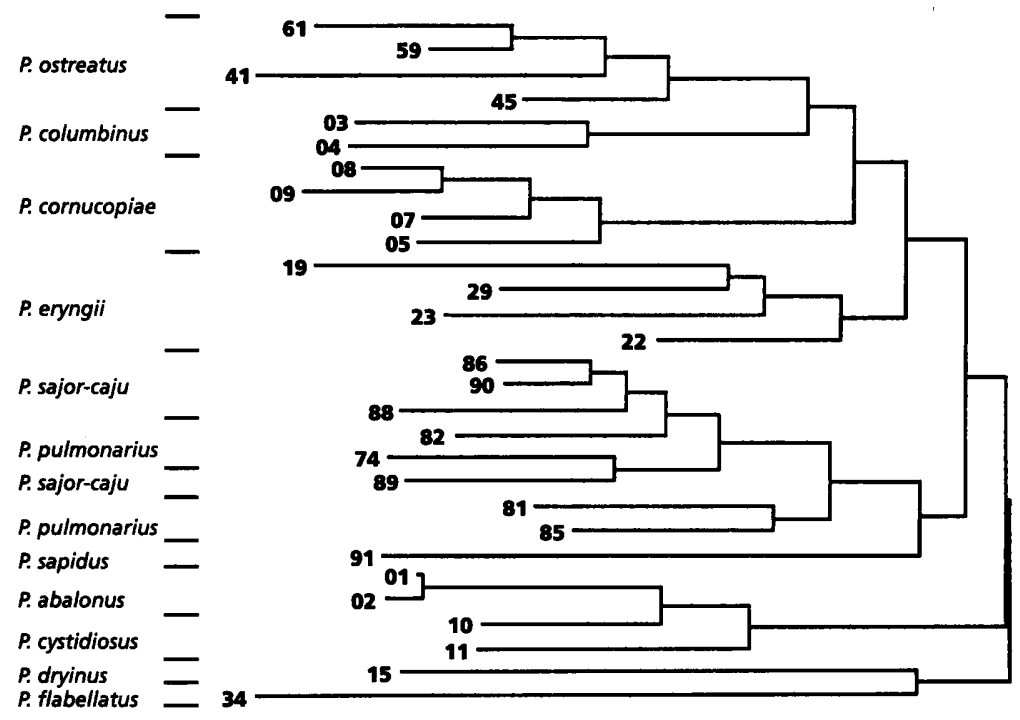

Fig. 3. Relationships among 29 Pleurotus isolates as visualized by two tree-making methods: (a) the UPGMA clustering method - the dashed line indicates the level of distance $(D=0.50)$ that permitted the classification of the Pleurotus isolates into nine clusters (A to 1$)$; (b) the neighbourjoining clustering method. Both dendrograms were computed with the use of a distance estimator (Nei \& Li, 1979). two isolates of $P$. abalonus presented five species-specific patterns (BamHI, ScaI, AvaI and SmaI) while P. cystidiosus strains presented identical phenotypes for SmaI.

\section{DISCUSSION}

Polymorphism in rDNA was scored in 29 isolates previously assigned to eleven Pleurotus species from various geographical areas throughout the world. All isolates have been previously studied and characterized by the use of morphological and physiological criteria (Zervakis \& Balis, 1991), and/or by isozyme analysis (Zervakis \& Labarère, 1992; Zervakis et al., 1994).

All the isolates had specific RFLP patterns and presented a high molecular polymorphism (with the sole exception of $P$. abalonus) comparable to the polymorphism evidenced by isozyme analysis (Zervakis \& Labarère, 1992; Iraçabal \& Labarère, 1993; Zervakis et al., 1994). In comparison with the DNA polymorphism of species of the order Agaricales previously described in the genera Armillaria (Anderson et al., 1987), Agaricus (Horgen et al., 1984; Hintz et al., 1989), Laccaria (Armstrong et al., 1989; Gardes et al., 1990) and Lentinus (Kulkarni, 1991), the rDNA polymorphism evidenced in the Pleurotus genus is higher. This could be attributed (i) to the efficiency of the probe used which was a part of the $P$. cornucopiae rDNA unit mainly constituted by intergenic sequences and (ii) to the loss or creation of new restriction sites and/or of length mutations as already evidenced in $P$. cornucopiae rDNA (Iraçabal \& Labarère, 1994). The probes used for other studies in Agaricales were either a heterologous rDNA or the entire rDNA unit consisting mostly of conserved sequences, apparently offering a less sensitive detection.

In genus Pleurotus, the rDNA probe showed higher polymorphism when DNA was digested by AvaI, ClaI, 
EcoRI or $S_{s t} \mathrm{I}$; the combination of two digestions (EcoRI with one of the endonucleases HindIII, KpnI, ScaI, SstI, SmaI, AvaI or ClaI) allowed discrimination of all isolates examined. It is of interest to note that the high variability evidenced in the EcoRI digestion as well as the low polymorphism exhibited in Bam HI and HindIII digestions were previously reported in other fungal genera (Kohn $e t$ al., 1988; Smith \& Anderson, 1989; Gardes et al., 1990; Kulkarni, 1991).

The ribosomal probe used allowed detection of fragments of high molecular size, whose size was larger than that estimated for the rDNA unit (over 10-12 kb). These fragments could correspond to: (i) rDNA fragments produced by partial digestions [this depends on phenomena affecting the endonuclease's specificity and digestibility according to reaction conditions (salt concentration, DNA purity, etc.) and to rDNA methylation (Buckner $e t$ al., 1988; Specht et al., 1984; Vilgalys \& Gonzalez, 1990; Li \& Horgen, 1993]; (ii) rDNA fragments located at the extremities of the rDNA cluster flanked by non-ribosomal sequences whose restriction sites differ according to species; (iii) non-ribosomal fragments possessing homology with some sequences of the probe. When these large fragments were omitted from the numeral analysis of RFLP patterns, interspecific relationships of Pleurotus species derived from the dendrograms based on all detected fragments were not affected; only minor changes were observed concerning intraspecific relationships.

Numerical treatment of RFLP data from the 29 Pleurotus strains was performed according to two tree-making methods (UPGMA and the neighbour-joining method), which showed single-species clusters for $P$. flabellatus, $P$. dryinus, $P$. sapidus and $P$. eryngii and two two-species clusters: $P$. abalonus $-P$. cystidiosus and $P$. pulmonarius $-P$. sajor-caju. One difference was that $P$. ostreatus, $P$. columbinus and $P$. cornucopiae were separated into three clusters by UPGMA analysis and joined into a common larger cluster by the neighbour-joining method. The species $P$. flabellatus, $P$. dryinus and, to a lesser degree, $P$. eryngii are clearly discriminated by RFLPs. This is in accordance with the anatomical and physiological characteristics (basidiocarp structure, presence of velum and formation of chlamydospores, host specialization, etc.) of these species, which permitted their assignment into discrete categories within the genus Pleurotus (Singer, 1986) and with the two previous isozyme analyses (Zervakis \& Labarère, 1992; Zervakis et al., 1994); these data indicate the suitability of using RFLPs in studies of fungal systematics.

The two Coremiopleurotus species ( $P$. abalonus and $P$. cystidiosus), which are grouped in cluster $C$, presented large phenetic distances with the other Pleurotus taxa examined; this is in accordance with their anatomical features, which are rather rare in the genus (production of synnematoid fructifications from the haploid and the dikaryotic mycelium) and with the previous isozyme data showing the clear separation of $P$. cystidiosus from other Pleurotus taxa (Zervakis \& Labarère, 1992). Although grouped in the same cluster, these two taxa had high phenetic diversity and seem to be quite unrelated to each other, suggesting that $P$. abalonus and $P$. cystidiosus evolved as discrete taxa.

The most unusual position of Pleurotus isolates in the RFLP dendrograms concerned the $P$. pulmonarius, $P$. sajorcaju and $P$. sapidus isolates. The mixed positioning of strains of the first two species in the same cluster accords with the numerous morphological similarities (Zervakis \& Balis, 1991), the significant interfertility (Hilber, 1982; G. Zervakis \& C. Balis, unpublished results) and the close proximity revealed from biochemical studies (Zervakis \& Labarère, 1992; Zervakis et al., 1994); this supports the hypothesis that $P$. sajor-caju is apparently a mistaken name to describe a Pleurotus taxon, as was previously used for a similar-looking Lentinus species ( $L$. sajor-caju Fr.). It seems that the isolates obtained for the purposes of this study, and assigned as $P$. sajor-caju, belong to an Asiatic population of $P$. pulmonarius. The four isolates (three European and one American) assigned as $P$. pulmonarius were clearly separated from the $P$. ostreatus and $P$. cornucopiae isolates; they probably coincide with the European and North American $P$. pulmonarius intersterility groups II, defined by Vilgalys $e t$ al. (1993). The single $P$. sapidus strain, although clearly separated from both these species, showed strong affinity with them; it was more related to $P$. pulmonarius than to $P$. sajor-caju, for which common morphological and physiological features have been described (Zervakis \& Balis, 1991). Positioning of the $P$. sapidus isolate was difficult. An isozyme study performed by starch-gel electrophoresis suggested, as did the RFLP data, more affinity of $P$. sapidus with $P$. pulmonarius than with $P$. ostreatus. Based on the closer relationships of the $P$. sapidus isolate with $P$. pulmonarius isolates, these isolates probably belong to the North American intersterility group II as defined by Vilgalys et al. (1993). Moreover, previous works based on morphological and compatibility criteria (Eger et al., 1979 ) or isozyme data (May \& Royse, 1988) suggested that $P$. sapidus was conspecific with $P$. ostreatus. The confusing positioning of the $P$. sapidus isolate could be attributed to the high heterogeneity of the $P$. sapidus group as pointed out by Hilber (1982).

By comparing the dendrograms from the two tree-making methods, only one difference was observed: P. ostreatus, $P$. columbinus and $P$. cornucopiae belonged to three clusters by UPGMA analysis and were joined into a common larger cluster by the neighbour-joining method. This can be explained by the fact that the neighbour-joining method minimizes the total branch length at each stage of clustering strains or groups of strains. However, the three species were clearly separated within the cluster, suggesting that they had a common ancestor rather than that they form a unique species. Indeed, $P$. cornucopiae presented distance values greater than $55 \%$ in all comparisons with other species, and was distinguished more readily from $P$. columbinus and $P$. ostreatus in both dendrograms. Previous systematic studies based on isozyme data also allowed $P$. cornucopiae to be separated clearly from the other Pleurotus species (Zervakis \& Labarère, 1992; Zervakis et al., 1994). In accordance with findings of morphological and compatibility studies 
(Bresinsky et al., 1977; Hilber, 1982) and of isozyme analyses (Zervakis \& Labarère, 1992; Zervakis et al., 1994), all these data are consistent with $P$. cornucopiae being a distinct species. Accordingly, the four European isolates assigned as $P$. cornucopiae certainly belong to the European group IV described by Vilgalys et al. (1993). Moreover, the $P$. ostreatus and $P$. columbinus species had large phenetic distances $(>52 \%)$ among their individual members. This induced Vilgalys et al. (1993) to define a European $P$. ostreatus group I, composed of both $P$. ostreatus and $P$. columbinus isolates. We presumed that our $P$. ostreatus and $P$. columbinus isolates, separated in two neighbouring clusters, could be associated with this European group I. However, the RFLP data confirmed recent biochemical studies (Zervakis \& Labarère, 1992; Zervakis et al., 1994) and demonstrated that, although possessing relationships, the $P$. ostreatus isolates studied were clearly separated from $P$. columbinus; these data suggested that $P$. ostreatus and $P$. columbinus are discrete taxa and have independent evolution processes. Furthermore, others (Hilber, 1982; Zervakis \& Labarère, 1992; Petersen \& Hughes, 1993; Vilgalys et al., 1993; Zervakis et al., 1994) have demonstrated that P. ostreatus, $P$. columbinus and $P$. cornucopiae are not related to $P$. pulmonarius, which was clearly separated from these species in spite of the conclusions drawn previously by Eger $e t a l$. (1979).

The close phenetic relationships as demonstrated by the relative position on the dendrograms of $P$. pulmonarius, $P$. sajor-caju and $P$. sapidus, and the clear separation of the $P$. flabellatus, $P$. dryinus and $P$. eryngii species are evidenced both by the present RFLP study and by previous works based on isozyme analysis (Zervakis \& Labarère, 1992; Zervakis et al., 1994). A similar phylogenetic congruence of data obtained from protein electrophoresis and DNA hybridizations has been reported for Agaricus bisporus (Royse \& May, 1982; Loftus et al., 1988) and Lentinus edodes (Royse et al., 1983; Kulkarni, 1991). Comparison of the Pleurotus systematics based on RFLP with previous isozyme data (Zervakis \& Labarère, 1992) showed that phenetic distances were greater when determined from zymograms; detection of more differences by isozyme analysis can be explained by the influence of environmental factors on protein conformation and activity, which constitute the major drawback in anatomical or biochemical studies.

In conclusion, systematic data based upon rDNA polymorphism analysis are in accordance with those based upon morphological and physiological criteria as well as with compatibility and biochemical methods; they furnish additional information on the relatedness and possible origins of individual species. Moreover, recovery of RFLP fragments (mainly HindIII and EcoRI fragments) characteristic of a species or of related species allowed five taxa, i.e. P. cornucopiae (2 fragments), P. ostreatus (2 fragments), $P$. abalonus (2 fragments), $P$. columbinus and $P$. sajor-caju, and two clusters, $C$ (P. abalonus and P.cystidiosus) and $\mathrm{E}(P$. sajor-caju and $P$. pulmonarius) to be distinguished, and to provide efficient molecular markers for a rapid identification of some species for a routine examination in
Pleurotus. These species-specific bands are of an indicative importance only, as a limited number of isolates per species were studied, but they could be used for further systematic studies on larger samples of Pleurotus.

\section{ACKNOWLEDGEMENTS}

We thank Professor C. Balis (Agricultural University of Athens) for kindly providing most of the Pleurotus isolates used in this study, and Professor J. Sourdis for his valuable contribution to the construction of the phylogenetic dendrograms. This work was supported by grants from the Conseil Scientifique de l'Université de Bordeaux II, the Institut National de la Recherche Agronomique, the Conseil Régional d'Aquitaine, the Lur Berri Food Company and an Erasmus-EEC fellowship.

\section{REFERENCES}

Anderson, J. B., Petsche, D. M. \& Smith, M. L. (1987). Restriction fragment polymorphisms in biological species of Armillaria mellea. Mycologia 79, 69-76.

Anderson, N. A., Wang, S. A. \& Schwandt, J. W. (1973). The Pleurotus ostreatus-sapidus species complex. Mycologia 65, 28-35.

Armstrong, J. L., Fowles, N. L. \& Rygiewicz, P. L. (1989). Restriction fragment length polymorphisms distinguish ectomycorrhizal fungi. Plant Soil 116, 1-7.

Bresinsky, A., Fischer, M., Meixner, B. \& Paulus, W. (1987). Speciation in Pleurotus. Mycologia 79, 234-245.

Bresinsky, A., Hilber, O. \& Molitoris, H. P. (1977). The genus Pleurotus as an aid for understanding the concept of species in Basidiomycetes. In The Species Concept in Hymenomycetes, Pp. 209-250. Edited by H. Clémençon. Vaduz: J. Cramer.

Buckner, B., Novotny, C. P. \& Ullrich, R. C. (1988). Organization of the ribosomal RNA genes of Schizopbyllum commune. Curr Genet 13, 417-424.

Carder, J. H. \& Barbara, D. J. (1991). Molecular variation and restriction fragment length polymorphisms (RFLPs) within and between six species of Verticillium. Mycol Res 95, 935-942.

Cassidy, J. R., Moore, D., Lu, B. C. \& Pukkila, P. J. (1984). Unusual organization and lack of recombination in the ribosomal RNA genes of Coprinus cinereus. Curr Genet 8, 607-613.

Chang, S. T. \& Miles, P. G. (1991). Recent trends in world production of cultivated mushrooms. Mushroom J 504, 15-18.

Eger, G., Li, S. F. \& Leal-Lara, H. (1979). Contribution to the discussion of the species concept in the Pleurotus ostreatus complex. Mycologia 71, 577-588.

Förster, H., Kinscherf, T. G., Leong, S. A. \& Maxwell, D. P. (1988). Estimation of relatedness between Pbytophthora species by analysis of mitochondrial DNA. Mycologia 80, 466-478.

Gardes, M., Fortin, A., Mueller, G. M. \& Kropp, B. R. (1990). Restriction fragment length polymorphism in the nuclear ribosomal DNA of four Laccaria spp: L. bicolor, L. laccata, L. proxima, and $L$. amethystina. Phytopathology 80, 1312-1317.

Gardes, M., Mueller, G. M., Fortin, A. \& Kropp, B. R. (1991). Mitochondrial DNA polymorphisms in Laccaria bicolor, L. laccata, L. proxima and L. amethystina. Mycol Res 95, 206-216.

Hilber, O. (1982). Die Gattung Pleurotus (Fr.) Kummer unter besonderer Berücksichtigung des Pleurotus eryngiiFormenkomplexes. Bibl Mycol 87, 1-448. Vaduz: J. Cramer.

Hintz, W. E., Anderson, J. B. \& Horgen, P. A. (1988). Physical mapping of the mitochondrial genome of the cultivated mushroom Agaricus brunnescens (= A. bisporus). Curr Genet 14, 43-49. 
Hintz, W. E., Anderson, J. B. \& Horgen, P. A. (1989). Relatedness of three species of Agaricus inferred from restriction fragment length polymorphism analysis of the ribosomal DNA repeat and mitochondrial DNA. Genome 32, 173-178.

Horgen, P. A., Arthur, R., Davy, O., Moum, A., Herr, F., Straus, N. \& Anderson, J. (1984). The nucleotide sequence homologies of unique DNAs of some cultivated and wild mushrooms. Can $J$ Microbiol 30, 587-593.

Iraçabal, B. \& Labarère, J. (1993). Comparison of polymorphism and phenetic variability as determined by the study of hydrolases and oxidoreductases in two cultivated mushrooms, Agaricus bisporus and Pleurotus cornucopiae. Exp Mycol 17, 90-102.

Iraçabal, B. \& Labarère, J. (1994). Restriction site and length polymorphism of the rDNA unit in the cultivated basidiomycete Pleurotus cornucopiae. Theor Appl Genet 88, 824-830.

Kistler, H. C., Bosland, P. W., Benny, U., Leong, S. \& Williams, P. H. (1987). Relatedness of strains of Fusarium oxysporum from crucifers measured by examination of mitochondrial and ribosomal DNA. Pbytopatbology 77, 1289-1293.

Kohn, L. M., Petsche, D. M., Bailey, S. R., Novak, L. A. \& Anderson, J. B. (1988). Restriction fragment length polymorphisms in nuclear and mitochondrial DNA of Sclerotinia species. Pbytopathology 78, 1047-1051

Kozlowski, M. \& Stépien, P. P. (1982). Restriction enzyme analysis of mitochondrial DNA of members of the genus Aspergillus as an aid in taxonomy. J Gen Microbiol 128, 471-476.

Kulkarni, R. K. (1991). DNA polymorphisms in Lentinula edodes, the shiitake mushroom. Appl Environ Microbiol 57, 1735-1739.

Li, A. \& Horgen, P. A. (1993). Evidence for cytosine methylation in ribosomal RNA genes and in a family of dispersed repetitive DNA elements in Agaricus bisporus and selected other Agaricus species. Exp Mycol 17, 356-361.

Loftus, M. G., Moore, D. \& Elliot, T.J. (1988). DNA polymorphisms in commercial and wild strains of the cultivated mushroom, Agaricus bisporus. Theor Appl Genet 76, 712-718.

Maniatis, T., Fritsch, E. F. \& Sambrook, J. (1982). Molecular Cloning: a Laboratory Manual. Cold Spring Harbor, NY: Cold Spring Harbor Laboratory.

May, B. \& Royse, D. J. (1988). Interspecific allozyme variation within the fungal genus Pleurotus. Trans Br Mycol Sor 90, 29-36.

Nei, M. \& Li, W. H. (1979). Mathematical model for studying genetic variation in terms of restriction endonucleases. Proc Natl Acad Sci US A 76, 5269-5273.

Noel, T. \& Labarère, J. (1989). Isolation of DNA from Agrocybe aegerita for the construction of a genomic library in Escherichia coli. Mush Sci 12, 187-201.

Petersen, R. H. \& Hughes, K. W. (1993). Intercontinental interbreeding collections of Pleurotus pulmonarius with notes on other species. Sydowia 45, 139-152.

Raper, J. R. \& Hoffman, R. M. (1974). Schizopbyllum commune. In Handbook of Genetics, pp. 597-626. Edited by R. C. King. New York: Plenum Press.

Rogers, S. O., Rehner, S., Bledsoe, C., Mueller, G. J. \& Ammirati, J. F. (1989). Extraction of DNA from Basidiomycetes for ribosomal DNA hybridizations. Can J Bot 67, 1235-1243.
Rohlf, F. J., Kishpaugh, J. \& Kirk, D. (1982). NT-SYS : Numerical Taxonomy System using Multivariate Statistical Programs. User Manual. SUNY at Stoney Brook, New York.

Royse, D. \& May, B. (1982). Genetic relatedness and its application in selective breeding of Agaricus brunnescens. Mycologia 74, 569-575.

Royse, D., Spear, M. \& May, B. (1983). Cell line authentication and genetic relatedness of lines of the shiitake mushroom, Lentinus edodes. J Gen Appl Microbiol 29, 205-216.

Saitou, N. \& Nei, M. (1987). The neighbor-joining method: a new method for reconstructing phylogenetic trees. Mol Biol Evol 4, 406-425.

Singer, R. (1986). The Agaricales in Modern Taxonomy. 4th edn. Germany: Koeltz Scientific Books.

Smith, M. L. \& Anderson, J. B. (1989). Restriction fragment length polymorphisms in mitochondrial DNAs of Armillaria: identification of North American biological species. Mycol Res 93, 247-256.

Sneath, P. H. \& Sokal, R. R. (1973). Numerical Taxonomy. The Principles and Practice of Numerical Classification. San Francisco: W. H. Freeman.

Sourdis, J. \& Nei, M. (1988). Relative efficiencies of maximum parsimony and distance matrix methods in obtaining the correct phylogenetic tree. Mol Biol Evol 5, 298-311.

Southern, E. (1975). Detection of specific sequences among DNA fragments separated by gel electrophoresis. J Mol Biol 98, 503-517.

Specht, C. A., Novotny, C. P. \& Ullrich, R. C. (1984). Strain specific differences in ribosomal DNA from the fungus Schizopbyllum commune. Curr Genet 8, 219-222.

Vilgalys, R. \& Gonzalez, D. (1990). Ribosomal DNA restriction fragment length polymorphism in Rbizoctonia solani. Pbytopatbology 80, 151-158.

Vilgalys, R., Smith, A., Sun, B. L. \& Miller, O. K. (1993). Intersterility groups in the Pleurotus ostreatus complex from the continental United States and adjacent Canada. Can J Bot 71, 113-128.

Vo-Quang, T., Malpiece, Y., Buffard, D., Kaminski, P. A., Vidal, D. \& Strosberg, A. D. (1985). Rapid large scale purification of plasmid DNA by medium or low pressure gel filtration. Application: construction of thermoamplifiable expression vectors. Biosci Rep 5, 101-111.

Walsh, S. R. A., Tyrrell, D., Humber, R. A. \& Silver, J. (1990). DNA restriction fragment length polymorphisms in the rDNA repeat unit of Entomophaga. Exp Mycol 14, 381-392.

Zervakis, G. \& Balis, C. (1991). Pleurotus species of Greece: an evaluation of their morphological and physiological characteristics. In Science and Cultivation of Edible Fungi, pp. 537-544 (part II). Edited by M. J. Maher. Rotterdam: Academic Press.

Zervakis, G. \& Labarère, J. (1992). Taxonomic relationships within the fungal genus Pleurotus as determined by isoelectric focusing analysis of enzyme patterns. J Gen Microbiol 138, 635-645.

Zervakis, G., Sourdis, J. \& Balis, C. (1994). Genetic variability and systematics of eleven Pleurotus species based on isozyme analysis. Mycol Res 98, 329-341.

Received 27 May 1994; revised 16 December 1994; accepted 2 February 1995. 